\title{
An Immersed-Boundary (IB)-Based 3D Tornado Model and Its Application
}

\author{
Xixiong Guo \\ Department of Mechanical and Industrial Engineering \\ Ryerson University \\ Toronto, Ontario, Canada, M5B 2K3 \\ xixiong.guo@ryerson.ca
}

\author{
Jun Cao \\ Department of Mechanical and Industrial Engineering \\ Ryerson University \\ Toronto, Ontario, Canada, M5B 2K3 \\ jcao@ryerson.ca
}

\begin{abstract}
A tornado features kinematically combining three major co-existent components, namely, updraft, translation, and rotation, which involve all the three dimensions in space while transient in time. For numerical simulation of a tornadobuilding interaction scenario, it looks quite challenging to seek a set of physically-rational and meanwhile computationallypractical boundary conditions to accompany traditional CFD approaches; thus, little literature can be found, as of today, in three-dimensional (3D) computational tornado dynamics study. Inspired by the development of immersed boundary (IB) method, this study employed the re-tailored Rankinecombined vortex model (RCVM) that applies the "relative motion" principle to the translational component of tornado, such that the building is viewed as "virtually" translating towards a "pinned" rotational flow that remains time-invariant at the far field region. This revision renders a steady-state kinematic condition applicable to the outer boundary of a large tornado simulation domain, successfully circumventing the boundary condition updating process that the original RCVM would have to suffer, and tremendously accelerating the computation. Here, this re-tailored RCVM was extended to its $3 \mathrm{D}$ version with the aid of logarithm law that describes the vertical flow evolution. Eventually, this tornado model was embedded in Incompact3D, an academic high-order finite difference turbulent flow solver, resulting in a practical powerful 3D tornado-building interaction simulation tool. A case study examined the tornadic wind induced loadings on a prismatic building; over all three directions, the vertical force component was found dominant, which effectively suggests the uprooting effect as observed in many reported scenes after a devastating tornado swept over.
\end{abstract}

Keywords: Re-tailored Rankine-combined vortex model (RCVM); Immersed boundary (IB); Large eddy simulation (LES); Finite difference method; Wind loadings.

\section{INTRODUCTION}

Tornadoes have been worldwide observed, and more frequently reported in United States and Canada. Compared to a straight-line wind, a tornadic flow is much more complex, since it is a type of airflow that essentially combines translational, vertical, and rotational velocities, and all components have to be considered during the investigation. Since the study of tornado dynamics through field observations or laboratory experiments tends to be time-consuming, laborious, and expensive, more research efforts have been made in the direction of mathematical modeling and numerical simulations. Historically, Wilson [1] first applied a two dimensional (2D) tornado model to examine the effects of tornadoes on rectangular-shaped buildings. Lewellen et.al. ${ }^{[2-3]}$ employed a three-dimensional (3D) large eddy simulation (LES) turbulence model to investigate the dynamics of a tornado-like vortex near the surface with particular attention paid to the turbulent flow characteristics in the corner region. Natarajan and Hangan ${ }^{[4]}$ also used LES model to study the effects of surface roughness and translation on the mean tangential velocity of the tornadic wind for different swirl ratios. On the other hand, tornado-induced wind loadings exerted on constructions have been also a practical topic. Based upon the Rankine-combined vortex model (RCVM), Selvam et.al. ${ }^{[5-6]}$ used the finite difference discretization of the NavierStokes (N-S) equations to conduct a series of studies focusing on the tornado-induced loadings on 2D sections of a cylinder and multi-cubic buildings and, more recently, some 3D cases ${ }^{[6]}$ at elevated Reynolds numbers also with the aid of LES ${ }^{[7]}$.

However, most aforementioned computational tornado dynamics studies mainly relied on conventional numerical methods, which need to update the time-dependent boundary conditions due to the dynamic nature of tornado. To overcome that tedious updating process, Guo et.al. ${ }^{[8-9]}$ re-formulated the RCVM to model the two-dimensional (2D) tornado-building interaction with the aid of immersed boundary (IB) method and the "relative motion" principle, resulting in a "virtual" scenario in which the building "moves" at a velocity that is the negative of the translation velocity of the tornado, while the background airflow has only a rotational component about its "pinned" center. Thus, as long as the computational domain is large enough for its outer boundary to little affected by the inner flow evolution induced by the "virtual translation" of the building, the time-dependency and, hence, the kinematic condition updating process, can be effectively eliminated at the outer boundary of the computational domain. 
This study aims to extend the $2 \mathrm{D}$ tornado simulation framework ${ }^{[8]}$ to the $3 \mathrm{D}$ version by taking the vertical velocity component into account. A combination of direct forcing IB approach with a centered finite difference scheme of high accuracy is performed. Meanwhile, the LES turbulence model is coupled with the N-S solver in order to better simulate the tornadic flow at relatively high Reynolds numbers. The advantage of this framework was discussed in terms of computational efficiency, accuracy, and simplicity [10]. Considering the large computational domain along with fine grid resolution required by $3 \mathrm{D}$ tornado simulations, this framework has been adapted to be portable on massive parallel computing platforms via Message Passing Interface (MPI) techniques ${ }^{[10]}$.

The rest of this paper will unfold as follows: the numerical method, including the tornado model, governing equations, and some other numerical treatment details, is presented in Section II. Section III provides a tornado-building interaction case with tornadic wind loadings analyzed in all directions. Finally, remarks are made in Section IV, addressing the conclusion and future extension of this study.

\section{NUMERICAL METHOD}

\section{A. Tornado Model}

The simplest model that can satisfy the Navier-Stokes equations while describing a tornadic wind field is the Rankine-combined vortex model (RCVM). In RCVM, tornado velocity profile can be decomposed into a constant-translation component, $\vec{V}_{t}$, a component of rotation with respect to the wind centre, $\vec{V}_{\theta}$, which denotes the tangential velocity component, and a vertical velocity component that obeys the logarithmic law to account for the boundary layer growth. The RCVM velocity profile along the radial direction from the tornado center consists of two distinct regions. The forcedvortex region refers to a circular area in which the radius from the tornado centre, $r$, is less than the $r_{c}$, the maximum radius of the forced-vortex region, $V_{\theta}$ varies linearly with $r$ and reaches the maximum tangential velocity at $r_{c}$. At radii larger than $r_{c}$, i.e., in the free-vortex region, $V_{\theta}$ decreases with the radius. The detailed mathematical expression of $V_{\theta}$ is as follows:

$$
V_{\theta}= \begin{cases}r \omega, & r \leq r_{c} \\ r_{c}^{2} \omega / r, & r>r_{c}\end{cases}
$$

where $\omega$ is the constant angular velocity. If taking the translational velocity and counter-clockwise rotational velocity into consideration, the resultant velocity on a horizontal plane can be re-projected to the Cartesian coordinate system, resulting in ${ }^{[5]}$ :

$$
u= \begin{cases}\left(V_{t}-y \cdot \omega\right) Z_{f}, & r \leq r_{c} \\ \left(V_{t}-y \cdot r_{c}^{2} \omega / r\right) Z_{f}, & r>r_{c}\end{cases}
$$

This study is fully funded by the Discovery Grant of the Natural Sciences and Engineering Research Council (NSERC) of Canada.

$$
v= \begin{cases}\left(\left(x+V_{t} \cdot t\right) \omega\right) Z_{f}, & r \leq r_{c} \\ \left(\left(x+V_{t} \cdot t\right) r_{c}^{2} \omega / r\right) Z_{f}, & r>r_{c}\end{cases}
$$

Remark that in Eqs. (2) and (3) $Z_{f}$ denotes a vertical kinematics adjustment parameter, which is a logarithmic law based function of vertical coordinate, $z$, as follows:

$$
Z_{f}=\frac{u^{*}}{k} \operatorname{In}\left(\frac{z+z_{0}}{z_{0}}\right)
$$

where $k$ and $z_{0}$ are constants, with $k=0.4, z_{0}=0.00375$, respectively. When using the re-tailored RCVM in which the rotational flow component is virtually viewed as pinned, the translational velocity $\vec{V}_{t}$ of the air vanishes, and Eqs. (2) and (3) reduce to:

$$
\begin{aligned}
& u= \begin{cases}(-y \cdot \omega) Z_{f}, & r \leq r_{c} \\
\left(-y \cdot r_{c}^{2} \omega / r\right) Z_{f}, & r>r_{c}\end{cases} \\
& v= \begin{cases}(x \cdot \omega) Z_{f}, & r \leq r_{c} \\
x \cdot\left(r_{c}^{2} \omega / r\right) Z_{f}, & r>r_{c}\end{cases}
\end{aligned}
$$

\section{B. Governing Equations}

The simulations are performed with Incompact $3 d$, an opensource code developed mainly by Laizet \& Lamballais ${ }^{[11]}$. All governing equations are solved on a collocated velocity grid via the six-order central compact finite different scheme ${ }^{[12]}$, while the pressure is on a staggered grid. A second-order Adams-Bashforth (AB) scheme is used for time-advancement and continuity is verified at the end of each sub-time step by solving a pressure Poisson equation. This Poisson equation is solved through a spectral solver to avoid the expensive cost incurred by the high-order discretization along with iterative schemes. The massively parallel version of the code was employed through an MPI implementation based on pencil domain decomposition strategy ${ }^{[10]}$.

The mass and momentum conservation principles are represented by the Navier-Stokes equations, which have the following form for an incompressible fluid:

$$
\begin{gathered}
\rho\left(\frac{\partial \vec{u}}{\partial t}+(\vec{u} \cdot \vec{\nabla}) \vec{u}\right)=-\vec{\nabla} p+\mu \vec{\nabla}^{2} \vec{u}+\overrightarrow{\mathrm{f}} \\
\vec{\nabla} \cdot \vec{u}=0
\end{gathered}
$$

where $\vec{u}(\vec{x}, t)=(u(\vec{x}, t), v(\vec{x}, t), w(\vec{x}, t))^{t}$ is the fluid velocity, $p(\vec{x}, t)$ is the pressure field, and $\rho$ is constant $(\rho=1)$ in the present framework. $\overrightarrow{\mathrm{f}}$ is the external forcing term caused by the interaction between the air and the immersed building.

\section{Time Advancement}

The time integration for solving Eq. (7) is performed using a second-order Adams-Bashforth scheme. A fractional step method ${ }^{[13]}$ is applied to decouple the velocity and pressure, and detailed steps are as follows: 


$$
\begin{aligned}
& \frac{\vec{u}^{*}-\vec{u}^{n}}{\Delta t}=\frac{3}{2} \vec{N}^{n}-\frac{1}{2} \vec{N}^{n-1}-\vec{\nabla} \tilde{p}^{n}+\tilde{\overrightarrow{\mathrm{f}}}^{n+1} \\
& \frac{\vec{u}^{* * *}-\vec{u}^{*}}{\Delta t}=\vec{\nabla} \tilde{p}^{n} \\
& \vec{\nabla} \tilde{p}^{n+1}=\frac{\vec{\nabla} \cdot \vec{u}^{* *}}{\Delta t} \\
& \frac{\vec{u}^{n+1}-\vec{u}^{* *}}{\Delta t}=-\vec{\nabla} \tilde{p}^{n+1}
\end{aligned}
$$

with

$$
\vec{N}^{n}=-\vec{u}^{n} \cdot \vec{\nabla} \vec{u}^{n}+\mu \vec{\nabla}^{2} \vec{u}^{n}
$$

and

$$
\tilde{p}^{n+1}=\frac{1}{\Delta t} \int_{t_{n}}^{t_{n+1}} p d t, \quad \tilde{\overrightarrow{\mathrm{f}}}^{n+1}=\frac{1}{\Delta t} \int_{t_{n}}^{t_{n+1}} \overrightarrow{\mathrm{f}} d t,
$$

where $\vec{u}^{* *}$ and $\vec{u}^{*}$ are the intermediate velocities obtained between time steps $n$ and $n+1$

\section{Evaluation of Forcing Term}

In order to solve the Eq.(9), the forcing term $\tilde{\overrightarrow{\mathrm{f}}}^{n+1}$ needs to be determined in advance. This term is generated by the IB approach, and reflects the interaction between the immersed object and the fluid. In this study, a direct method is adopted to calculate, the forcing term as follows:

$$
\tilde{\overrightarrow{\mathrm{f}}}^{n+1}=\varepsilon\left(-\frac{3}{2} \vec{N}^{n}+\frac{1}{2} \vec{N}^{n-1}+\vec{\nabla} \tilde{p}^{n}+\frac{\vec{u}_{0}^{n+1}-\vec{u}^{n}}{\Delta t}\right)
$$

with $\varepsilon=1$ in the solid body region, and $\varepsilon=0$ elsewhere else in the computational domain. The target velocity $\vec{u}_{0}$ is calibrated in order to satisfy the no-slip condition at the surface of solid body.

\section{E. Entire Procedure}

Details about the spatial discretization using sixth-order compact finite difference method can be found in ${ }^{[10]}$. Here, a typical solution procedure for each time step within the simulation framework is described as follows:

1. Set up a computational domain, and initialize the velocity field using the RCVM as aforementioned in Eqs. (5) and (6);

2. Based on the location of the boundary of the immersed body at time step $n+1$, identify the fluid, solid and solid boundary points on the Eulerian grid;

3. Calculate the target velocity $\vec{u}_{0}^{n+1}$ in Eq.(15);

4. Obtain the forcing term using Eq.(15);

5. Compute the velocity field $\vec{u}^{*}$ by solving Eq.(9);

6. Compute intermediate velocity $\vec{u}^{* *}$ by solving Eq.(10);

7. Compute the pressure $\vec{\nabla} \tilde{p}^{n+1}$ by solving pressure Poisson equation Eq.(11);

8. Correct divergence-free fluid velocity $\vec{u}^{n+1}$ at the $(n+1)^{\text {th }}$ time step by solving Eq. (12);

9. Go back to Step 2 if time-marching is still needed; otherwise, stop the computation.

\section{NUMERICAL RESULTS}

\section{A. Initial condition and Boundary condition}

As aforementioned in the beginning of the precedent section, this study re-tailored the original RCVM, such that the scenario of a tornado passing through a building (prism) is interpreted as a superposition of a "pinned" rotational airflow and a "virtual translation" of the building, which is opposite to the physical translation direction of the tornado. Then, the retailored RCVM serves to set up the initial velocity background, and note that the outer boundary condition has been rendered time-independent owing to this re-tailored model.

Figure 1 illustrates the computational domain with $\left(\mathrm{L}_{\mathrm{x}}, \mathrm{L}_{\mathrm{y}}\right.$, $\left.\mathrm{L}_{\mathrm{z}}\right)=(24,16,16)$. The length (in the $x$-direction) and the height (in the $z$-direction) of the prism are equal to 1 , which is equal to the radius of the forced vortex region, $r_{c}$, and the depth (in the $y$-direction) of prism is set at 12 . The translation velocity remains one unit. The Reynolds number based on the height of the prism is $\operatorname{Re}=10000$. No slip boundary condition is applied on the bottom surface, as well as the prism walls, and the velocity on all other surfaces is governed by the re-tailored time-independent RCVM.

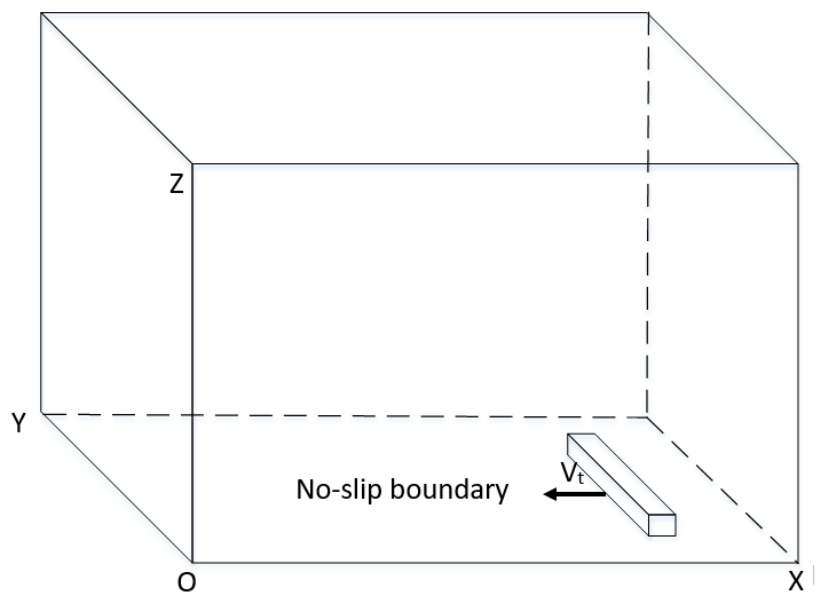

Fig.1 Schematic of the computational domain

\section{B. Force Coefficient Definition}

The dimensionless force coefficients in all three directions are defined as:

$$
\begin{aligned}
& C_{x}=\frac{F_{x}}{0.5 \rho V_{t}^{2} S} \\
& C_{y}=\frac{F_{y}}{0.5 \rho V_{t}^{2} S} \\
& C_{z}=\frac{F_{z}}{0.5 \rho V_{t}^{2} S}
\end{aligned}
$$

where $F_{x}, F_{y}, F_{z}$ and $S$ is the components of the resultant force in three directions and projection area, respectively. Using the 
$x$-direction as example, $F_{x}=-\int_{\Omega_{0}} f_{1} d \mathbf{x}$, and $f_{1}$ is the $x$ component of the force density, the calculation of which is based upon the following integral form of the $x$-component of the momentum equations on any fluid domain $\Omega_{0}$ :

$$
\begin{aligned}
& \frac{\partial}{\partial t} \int_{\Omega_{0}} \rho u_{1} d \mathbf{x}+\int_{\partial \Omega_{0}} \rho u_{1} \mathbf{u} \cdot \mathbf{n} d s \\
& =-\int_{\Omega_{0}} p n_{1} d \mathbf{x}+\int_{\partial \Omega_{0}} \mu\left(\frac{\partial u_{1}}{\partial x_{j}}+\frac{\partial u_{j}}{\partial x_{1}}\right) n_{j} d s+\int_{\Omega_{0}} f_{1} d \mathbf{x}
\end{aligned}
$$

The computations of $F_{y}, F_{z}$ are similar to $F_{x}$.

\section{Case Study}

In this case, the initial position of the prism is placed at 3 units from right boundary of the computational domain at time step $\mathrm{t}=0$, as shown in Fig. 1 . When employing the re-tailored RCVM, the building is "virtually moving" towards left, while the tornado central vertical line remains located at the center of the computational domain. Fig. 2 illustrates the evolution of the force coefficients in all three directions $C_{x}, C_{y}$, and $C_{z}$. Note that the horizontal coordinate, $X$, is arranged such that, while $\mathrm{X}<0, \mathrm{X}=0$ and $\mathrm{X}>0$, the tornado center physically approaches the prism, nominally coincides with the prism, and leaves the prism, respectively.

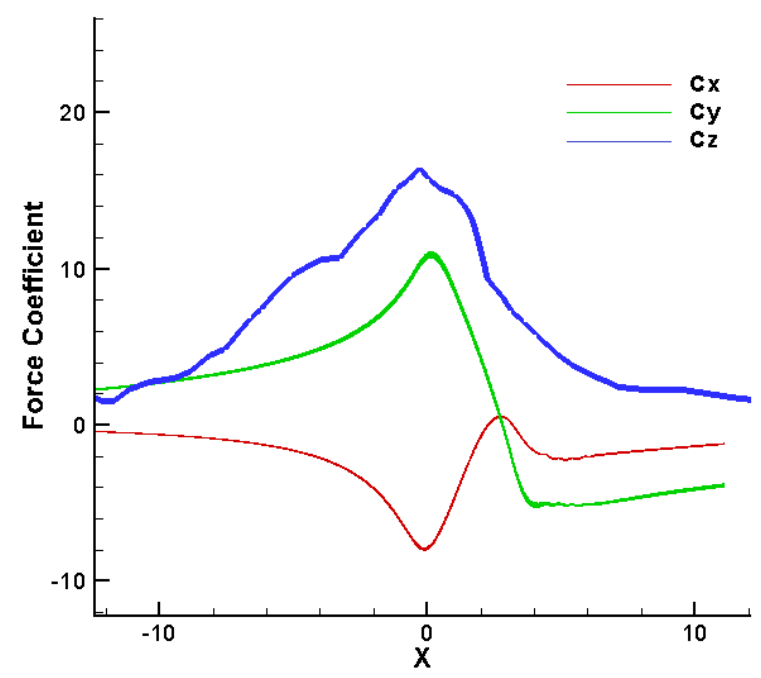

Fig.2 Evolution of force coefficients in three directions

When the translating tornado center approaches the prism, $C_{x}$ keeps decreasing and stays negative, while both $C_{y}$ and $C_{z}$ keep positive and continuously go up. This indicates the following tornado characteristics in the approaching stage: (1) the building is attracted by the tornado in the $x$-direction, and the closer between the tornado center of the building, the larger attractive force; (2) the building is pushed by the tornado towards the positive $y$-direction due to the counter-clockwise rotation direction of the airflow, and the closer between the tornado center of the building, the larger pushing force; and (3) the building experiences an upward suction force in the $z$ direction, and the closer between the tornado center of the building, the larger suction force.
When the tornado is in the stage of nominally coinciding with the building, the three force coefficients consistently reach their respective extreme values, challenging the wind-resistant capability of the building to the utmost extent.

Afterwards, in the stage of tornado leaving the building, the three force components are consistent, again, in exhibiting their magnitude decreases. However, the decreasing trend for the magnitude of $C_{z}$ remains unchanged all the way in this stage, tending to vanish at the end; on the other hand, $C_{x}$ and $C_{y}$ experience up and down in terms of their magnitudes. In detail, the $C_{x}$ magnitude drops until it reaches its second extreme, which is numerically very close to zero and, locally, the tornado center is about 3 units away from the building, then rerises during the period in which the distance between the tornado center and the building goes approximately from 3 units to 5 units, and then re-drops and gradually diminishes at the end; for the $C_{y}$ magnitude in this stage, it steeply drops and goes to zero when $C_{x}$ reaches its second extreme (at about $\mathrm{X}=3$ ) and, then, the direction of $F_{y}$ changes to the negative $y$ direction, and the $C_{y}$ magnitude remains increasing until approximately $\mathrm{X}=4$, followed by a decreasing trend. The complex up-and-down trend for $C_{x}$ and $C_{y}$ is attributed to the strongly disturbed wake behind the building after the tornado sweeps it over. The three force coefficients calm down only after the core zone of the tornado has been completely away from the building.

Compared with each other, the magnitude of the $z$-direction force component is the highest, suggesting that the influence caused by the given logarithm law based vertical adjustment parameter $Z_{f}$ is a dominant factor for the tornado dynamics study.

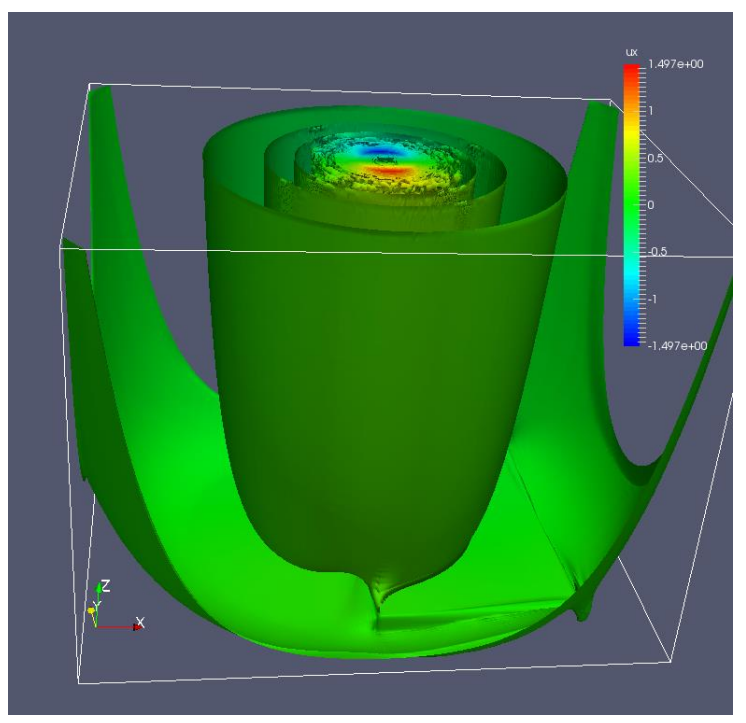

Fig.3 Velocity profile when tornado center coincides with prism

Figure 3 provides a $3 \mathrm{D}$ view of tornadic flow when the tornado center coincides with the building. A number of eddies are found in the tornado core area on a horizontal section near the top face of the computational domain, while beyond the core zone the streamlines overall remain circular about the center of tornado. Figure 4 zooms into the vicinity of the 
building, indicating the no-slip condition on the surfaces of the prism are satisfactorily guaranteed as no penetration is observed across the interface of the prism and air.
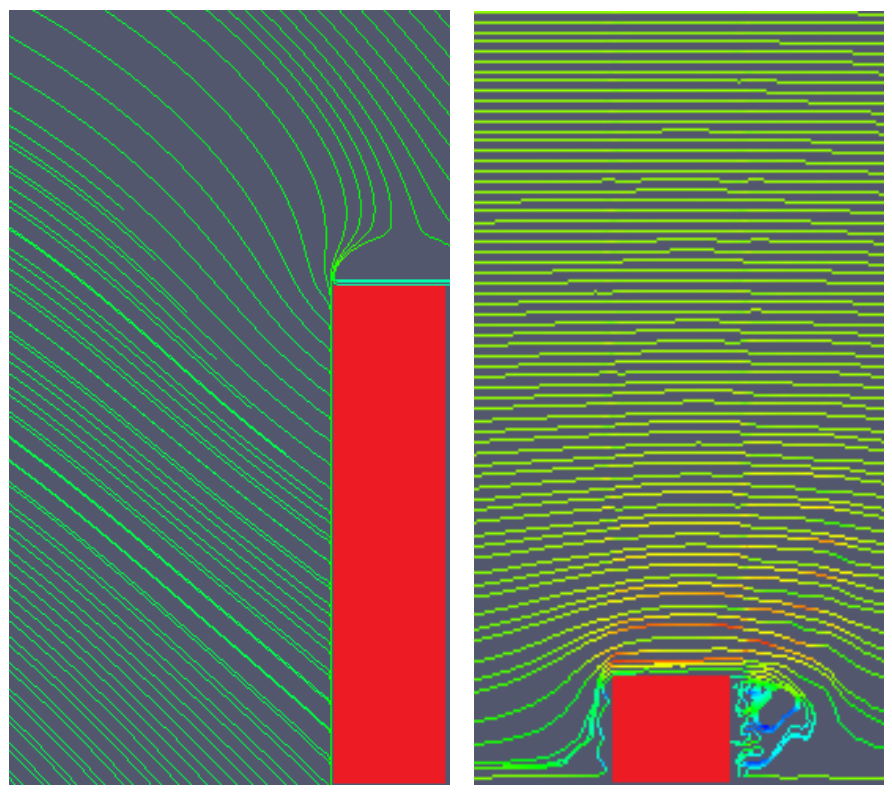

Fig.4 Streamlines near the prism on a horizontal $(x-y)$ and a vertical $(x-z)$ sections

\section{CONCLUSIONS}

In this paper, a novel computational framework was established, aiming at extending previously built 2D tornado simulation model to its $3 \mathrm{D}$ version by introducing a vertical kinematics adjustment parameter, $Z_{f}$, for describing the boundary layer growth in the $z$-direction. The re-tailored Rankine-Combined Vortex Model (RCVM) was employed with the aid of the immersed boundary (IB) approach, so that the numerical investigation of tornado-construction interaction can be made without requesting kinematic condition updating process at the outer boundary of the 3D computational domain. The results obtained in the present study has shown that the vertical component of a tornado plays a significant role in the tornado dynamics study since the largest force coefficient was found in the $z$-direction. Extensive simulations are underway to investigate the interaction between a variety of tornadic wind scenarios and differently deployed multi-constructions owing to the embedment of the IB strategy.

\section{ACKNOWLEDGMENT}

In addition to the Discovery Grant from the Natural Sciences and Engineering Research Council (NSERC) of Canada for the present study, the authors also wish to thank the Compute Canada for providing the computational facilities in support of the computations reported here.

\section{REFERENCES}

[1] N. B. Ward. The exploration of certain features of tornado dynamics using a laboratory model. Journal of the Atmospheric Sciences, 1972, 29, 1194-1204.

[2] W. S. Lewellen, D. C. Lewellen, R.I. Sykes. Large-eddy simulation of a tornado's interaction with the surface. Journal of the Atmospheric Sciences, 1997, 54(5): 581-605.

[3] D. C. Lewellen, W. S. Lewellen. Near-surface intensification of tornado vortices. Journal of the Atmospheric Sciences, 2007, 64(7): 2176-2194.

[4] D. Natarajan, H. Hangan. Large Eddy Simulation of Translation and Surface Roughness Effects on Tornado-like Vortices. Journal of Wind Engineering Applications and Industrial Aerodynamics, 2012, 104, 577 584.

[5] R. P. Selvam, P. C. Millett. Computer modeling of tornado forces on buildings. Wind and Structures, 2003, 6(3):209-220.

[6] P. Gorecki, R. P. Selvam. Rankine combined vortex interaction with a rectangular prism. International Journal of Computational Fluid Dynamics, 2015, 29(1): 120-132.

[7] J. Smagorinsky. General circulation experiments with the primitive equations: I. The basic experiment*. Monthly weather review, 1963, 91(3):99-164.

[8] X. Guo, J. Cao. An IB-LBM investigation into the aerodynamic coefficients in relation to the rotation intensity of a tornado-like wind. Computers and Mathematic with Applications, 2016, doi: 10.1016/j.camwa.2016.07.016 (In press).

[9] X. Guo, R. Palanisamy J. Cao. A two-dimensional IB-LBM framework combined with re-tailored RCVM for assessing the rotation intensity of a tornadic wind over a building configuration. Engineering Structures, 2017, 131, 57-68.

[10] S. Laizet S, N. Li. Incompact3d: a powerful tool to tackle turbulence problems with up to $\mathrm{O}\left(10^{5}\right)$ computational cores. International Journal for Numerical Methods in Fluids. 2011, 67(11),1735-57.

[11] S. Laizet, E. Lamballais. High-order compact schemes for incompressible flows: A simple and efficient method with quasi-spectral accuracy. Journal of Computational Physics, 2009, 228(16),5989-6015.

[12] S. K. Lele. Compact finite difference schemes with spectral-like resolution. Journal of Computational Physics, 1992, 103, 16-42.

[13] J. Kim, P. Moin. Application of a fractional-step method to incompressible Navier-Stokes equations. Journal of Computational Physics, 1985,59, 308-323. 